\title{
SPACEBORNE SAR IMAGERY STEREO POSITIONING BASED ON RANGE-COPLANARITY EQUATION
}

\author{
C.Q.Cheng ${ }^{\mathrm{a},}{ }^{*}$, J.X.Zhang ${ }^{\mathrm{a}}$, G.M.Huang ${ }^{\mathrm{a}}$,C.F.Luo ${ }^{\mathrm{a}}$ \\ ${ }^{\text {a }}$ Chinese Academy of Surveying and Mapping, Beijing 100039, China-cspring@casm.ac.cn
}

Commission VII

KEY WORDS: rigorous positioning, side looking radar, R-Cp equation, exterior orientation elements, SAR

\begin{abstract}
:
Stereo positioning is a important content for Spaceborne SAR imagery mapping, which is still lack of investigation depend on exterior orientation elements. In this paper, we firstly introduced the Range-Coplanarity (R-Cp) geometric imaging equation and its character for radar imagery, compared the difference and relationship between R-Cp and R-D model, and build stereo positioning rigorous model for spaceborne SAR imagery based on the R-Cp equation, satellite orbit, and pose refinement model. Then separate taking TerraSAR-X images in mountainous terrain observed from same and opposite side-looking direction as test data, we presented the initial attitude value acquirement method for TerraSAR-X image after moving compensation, analyzed stereo positioning precision according to amounts and spatial distributions of GCPs and lengths of baseline between SAR antennas, and also compared the precision with R-D model. the proposed stereo positioning model avoids SAR signal imaging parameters during the geometric processing, with one GCP it could improved the plan and height precision from $(17.9 \mathrm{~m}, 29.2 \mathrm{~m})$ to $(10.3 \mathrm{~m}, 6.1 \mathrm{~m})$ in the test of opposite side-looking direction images, and even had a better accuracy than R-D model when more GCPs were used, which indicate that R-Cp model have a potential application in spaceborne SAR image stereo positioning.
\end{abstract}

\section{INTRODUCTION}

The rigorous geometric imaging model of side-looking radar image mainly includes the range doppler equation and sensor exterior orientation elements. Leberl $\mathrm{F}$ model proposed by Leberl F (Leberl, 1978, 1990), according to range condition and zero doppler condition, complies with the radar imaging mechanism. The R-D model, with similar mechanism of Leberl F model, takes into account of the non-zero value situation of doppler frequency, and therefore has explicit geometric and physical meaning (Johnsen, et al., 1995). There have two kinds of model based on exterior orientation, the radar collinearity equation-based model(Konecny and Schuhr, 1988), is the extension of the application of collinearity equation in optical remote sensing image. The radar range coplanarity equation(Cheng et al, 2012),build according range condition and radar beam center coplanarity condition.

Currently, the SAR image geometric correction under different conditions has been more extensively studied (Lauknes,2004,

Johnsen,1995; Gelautz,1998). The stereo positioning when the 1960s was literature related to (La Prade,1963), However, due to the lack of suitable stereo image data, related research was unable to started, this situation changed when the RADARSAT launched in 1995, and most of SAR image positioning process adopted the R-D model. The stereo positioning research result used the elements of exterior orientation as positioning parameters still very scarce. As the Range-Coplanarity equation use the elements of exterior orientation as a directional parameters, the research of spaceborne SAR stereo positioning of sparse control point based on the R-Cp equation is significative.

In 2005, China launched the 1:50,000 mapping project in western difficult region where without the topographic map ever before, for example the Hengduan Mountains, Yunnan and Guizhou Plateau are covered by snow or fog all the year round, optical remote sensing images are difficult to separately complete the mapping tasks, coupled with ruggedness terrain in these areas, field surveying of ground control points is very difficult, so mapping with high-resolution SAR images for these specific regions is an important part for the project, also the spaceborne SAR images stereo mapping under the conditions of sparse control point is a key technology for this project. In this case, the paper based R-Cp equation research the TerraSAR-X images stereo positioning models, techniques and methods.

\section{THE STEREO POSITIONING MODEL BASED ON R-CP EQUATION}

\subsection{Introduction of R-Cp geometry imaging model}

R-Cp geometric imaging equation based on exterior elements is built through two conditions as following and its principle is shown as Fig 1(Cheng et al, 2012):

(1)All ground points corresponding to one line in image and the radar antenna at shoot time are within one center plane of radar beam, which is determined by state vector and attitude of

\footnotetext{
* Corresponding author.
} 
the sensor; (2) The spatial range between sensor and ground points is equal to the range measured by radar wave. The Coplanarity equation is build according to the fact that radar beam plan is perpendicular to $\mathrm{x}^{\prime}$ axes of satellite body coordinate system which is changing with attitude angles. Range-coplanarity condition is established by the following equations:

$$
\left\{\begin{array}{c}
R=|\overrightarrow{O P}-\overrightarrow{O S}| \\
\overrightarrow{\boldsymbol{i}} \cdot(\overrightarrow{O P}-\overrightarrow{O S})=0
\end{array}\right.
$$

$\overrightarrow{\boldsymbol{i}}$ is the normal vector of the plane of radar beam center (the same as $x^{\prime}$ axis which is converted from $x$ axis in the sensor coordinate system by rotating attitude angles), $\overrightarrow{\boldsymbol{O P}}, \overrightarrow{\mathrm{OS}}$ are the position vectors of ground points and the radar antenna, respectively. $R$ is the slant distance.

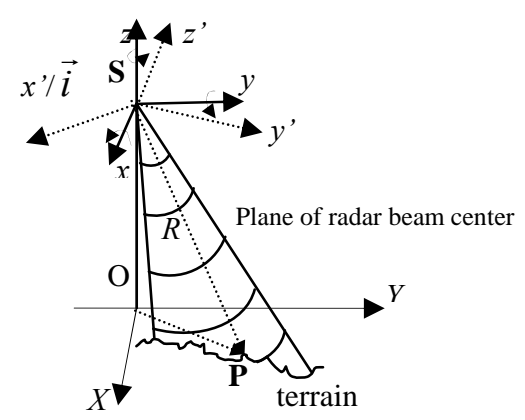

Fig 1. Range-coplanarity imaging geometry for side-looking radar

When the rotation of attitude angles of $\varphi-\kappa-\omega$ system is used, the expansion equation of imaging equation for radar image of planar scanning mode is correspondingly obtained as follow:

$$
\left\{\begin{array}{c}
(X-X s)(\cos \varphi \cos \kappa)+(Y-Y s) \sin \kappa-(Z-Z s)(\sin \varphi \cos \kappa)=0 \\
(X-X s)^{2}+(Y-Y S)^{2}+(Z-Z s)^{2}=\left(y M_{r}+R_{0}\right)^{2}
\end{array}\right.
$$

In formula(2), (Xs,Ys,Zs) is sensor location coordinate, $(\mathrm{X}, \mathrm{Y}, \mathrm{Z})$ is ground point coordinate, $M_{r}$ is the across-track resolution; $R_{0}$ is nearest slant distance; $y$ is the column coordinate of the pixel in the image.

For satellite radar image, paper (Cheng,2012) has given two kinds of R-Cp model in ECR coordinate system, one is:

$$
\left\{\begin{array}{c}
i_{X}(X-X s)+i_{Y}(Y-Y s)+i_{Z}(Z-Z s)=0 \\
(X-X s)^{2}+(Y-Y s)^{2}+(Z-Z s)^{2}-\left(y M_{r}+R_{0}\right)^{2}=0
\end{array}\right.
$$

The other kind is explicit function of image coordinate $(x, y)$ in ECR:

$$
\left\{\begin{array}{c}
x=\left[i_{X} \cdot(X-X s)+i_{Y} \cdot(Y-Y s)+i_{Z} \cdot(Z-Z s)\right] / M_{a}=0 \\
y=\left[\sqrt{(X-X s)^{2}+(Y-Y S)^{2}+(Z-Z s)^{2}}-R_{0}\right] / M_{r}
\end{array}\right.
$$

where , $\quad \vec{i}=\left[\begin{array}{lll}i_{X} & i_{Y} & i_{Z}\end{array}\right]^{T}=R_{O}{ }^{E} R_{b}{ }^{O}\left[\begin{array}{lll}1 & 0 & 0\end{array}\right]^{T}, R_{O}{ }^{E}, R_{b}{ }^{O}(\varphi, \kappa)$ are translate matrix special from orbit coordinate system to ECR coordinate system and from satellite body coordinate system to orbit coordinate system. formula(4) is suitable for combined adjustment for multi-source data than formula(3).

\subsection{The connection and difference between $\mathrm{R}-\mathrm{Cp}$ model and R-D model}

The Range-Doppler (R-D) model is the most widely used physical sensor model for spaceborne SAR remote sensing system(Curlander and McDonough, 1991). In the R-D model, the geometric characteristics of SAR imaging procedure are rigorously described by two equations in azimuth and range (Curlander,1982):

$$
\left\{\begin{array}{c}
R=|\overrightarrow{O P}-\overrightarrow{O S}| \\
f_{D}=2(\overline{V S}-\overrightarrow{V p}) \cdot(\overrightarrow{O P}-\overline{O S}) /(\lambda R)
\end{array}\right.
$$

where $f_{\mathrm{D}}$ is the Doppler frequency, $\lambda$ is the radar wavelength, $\overline{V S}$ and $\overline{V p}$ represents velocity vectors of SAR sensor and target respectively.

We can see from the range equation that it is the spatial point set, which the distance is $\mathrm{R}$ from ground points to sensor, and the geometric shape is range sphere; The doppler equation is a cone over the sensor; The coplanarity equation shows the ground points and the sensor in the same plane. On a sampling point, the geometry figure of R-D equation and R-Cp equation are shown in the left part of Figure (2).

For any radar image pixel we can calculate the doppler frequency of corresponding ground point, the distance between ground point and sensor antenna of photography time and the attitude values, then the R-D and R-Cp model can be built, which together with Earth ellipsoid model and the ground elevation model could process the radar image direct positioning. The intersection of the Earth ellipsoid equation with range equation, coplanar equation, or doppler equation separately presented on ground are a circle, an arc/line, and a hyperbolic. Therefore, the hyperbolic and circle intersection point on earth surface is the solution of the R-D model positioning, the arc/line and circle intersection point is the solution of positioning point under the R-Cp model, the principles of two models shown in the right part of Figure (2).

Figure 2 also shows the link and difference when the R-Cp model and R-D model positioning, and also concluded that different pixel points at the same image line for non sidelooking radar image with one group attitude values but different doppler frequency values. For the real aperture radar, when the precision sensor attitudes and the doppler frequency are known, the positioning result of R-D and R-Cp model is same. But for SAR images, because of its variety of imaging modalities, yet not one rigorous positioning model is common to all the images. If SAR imagery has the same features of geometric deformation as that of the image shoot by the real aperture radar under the ideal motion state referenced by the motion compensation process, the real aperture radar-based R$\mathrm{Cp}$ model established can still be useful to the SAR images. If 
in the process of focus on imaging caused other deformation, $\quad$ R-Cp model may not be suitable for.

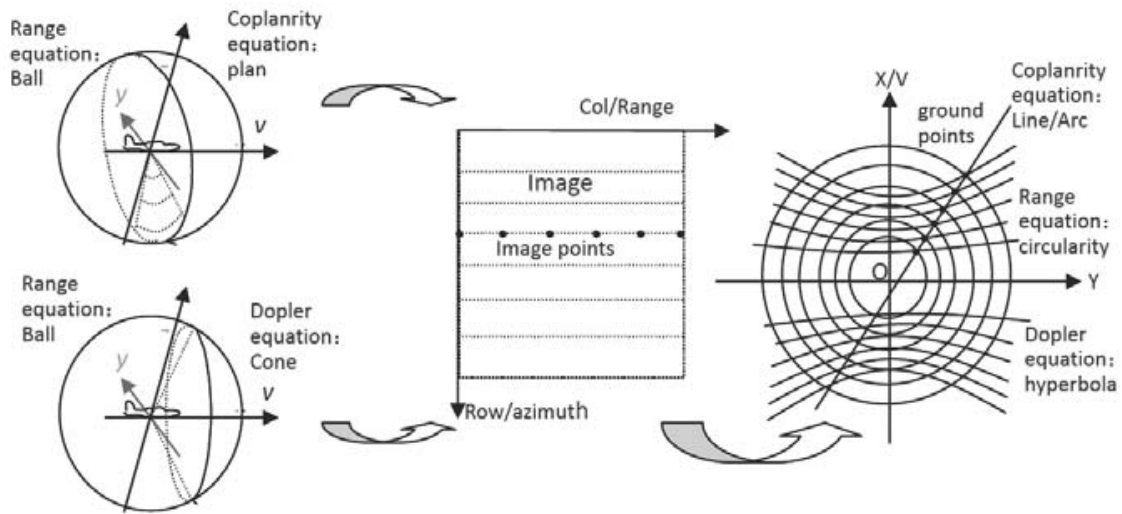

Fig 2 R-D model and R-Cp model positioning principle

\subsection{Orbit attitude model of spaceborne SAR}

In SAR imaging process, the motion compensation and focusing were occurred, so the satellite orbit and attitude data were compensated to an ideal state. Therefore, no matter what rigorous model, SAR remote sensing image geometry processing should use the data that motion compensation referenced. As the stability of the satellite in orbit, under normal circumstances, when imaging the reference orbit, the speed and the actual track, speed is approximately the same. Reference attitude is generally constant or uniform changes, in the absence of a reference value then the middle or mean value of original data could be taken as the initial value, and could get according to the Doppler parameter value.

TerraSAR-X image adopt the 0 value Doppler imaging parameters for focus, this means that the beam center planarity is perpendicular to the velocity direction in imaging, in this paper, according to this conditions to calculate the sensor initialization attitude value after SAR imaging motion compensation. When the three attitude angles are all zero, the beam center plane is perpendicular to the axis $\mathrm{X}_{\mathrm{O}}$ vector, when the Doppler parameter is 0 , the beam center plane is perpendicular to the velocity vector. $\mathrm{X}_{\mathrm{O}}$ axis and speed direction can achieve the coincidence by rotating around the $\mathrm{Y}_{\mathrm{O}}$-axis of $\varphi_{0}$ angle, therefore, when the Doppler parameter is 0 , equivalent to the three original attitude value is $\left(0, \varphi_{0}, 0\right)$. As a result, unit speed vector in geocentric rectangular coordinate could also be calculated by a unit vector $X \mathrm{o}$ axis and associated rotation matrix , that is:

$$
\left[\begin{array}{lll}
v_{x} & v_{y} & v_{z}
\end{array}\right]^{T}=R_{o}^{E} R_{r_{o}}\left(\varphi_{0}\right)\left[\begin{array}{lll}
1 & 0 & 0
\end{array}\right]^{T}
$$

$\left[v_{x}, v_{y}, v_{z}\right]^{\mathrm{T}}$ is unit speed vector. Based on the formula (6), $X_{\mathrm{O}}, Y_{\mathrm{O}}, Z_{\mathrm{O}}, V_{\mathrm{X}}, V_{\mathrm{Y}}, V_{\mathrm{Z}}$ is the satellite position and speed state vector in ECR coordinate system, the pitch angle $\varphi_{0}$ could be calculated:

$$
\varphi_{0}=-\operatorname{asin}\left[(Z)_{X} \cdot v_{x}+(Z 0)_{Y} \cdot v_{y}+(Z)_{Z} \cdot v_{z}\right]
$$

As the satellite's orbit and attitude in a certain time with very good stability, errors of initial value of orbit and attitude in a certain time expressed as systemic, and its orbit error and attitude error with low-level polynomial fitting could meet the positioning accuracy. For one scene the TerraSAR-X imaging track is short, linear polynomial is used as refined model for orbit attitude model, namely:

$$
\left\{\begin{array}{c}
X_{S}=X_{S 0}+a_{0}+a_{1} t \\
Y_{S}=Y_{S 0}+b_{0}+b_{1} t \\
Z_{S}=Z_{S 0}+c_{0}+c_{1} t \\
\varphi=\varphi_{0}+f_{0}+f_{1} t \\
\kappa=\kappa_{0}+g_{0}+g_{1} t
\end{array}\right.
$$

In the formula (8) $\left(X_{S 0}, Y_{S 0}, Z_{S 0}, \varphi_{0}, \kappa_{0}\right)$ are the ephemeris attitude initial value, $a_{i}, b_{i}, c_{i}, f_{i}, g_{i}(i=0,1 \ldots)$ are ephemeris and attitude refined model parameters.

\subsection{Stereo positioning model of spaceborne SAR}

In order to achieve the high accuracy of the remote sensing image positioning the orbital and attitude parameters must be refined. Substituted the linear model (8) into formula (4), the refined orbit attitude model parameters $a_{i}, b_{i}, c_{i}, f_{i}, g_{i}(i=1,2)$ and the ground point coordinates increment ( $\triangle X, \Delta Y, \Delta Z$ ) as the unknowns, coordinates measurement error equation of the image point are got through linearized R-Cp equations:

$$
\left\{\begin{array}{l}
v_{x}=f_{1 a 0} a_{0}+f_{1 b 0} b_{0}+f_{1 c 0} c_{0}+f_{1 f 0} f_{0}+f_{100} g_{0} \\
+f_{1 a l} a_{1}+f_{1 b b} b_{1}+f_{1 c 1} c_{1}+f_{1 f 1} f_{1}+f_{1 g 1} g_{1}-f_{1 X} \Delta X-f_{1 Y} \Delta Y-f_{1 Z} \Delta-l_{1} \\
v_{y}=f_{2 a 0} a_{0}+f_{2 b b} b_{0}+f_{2 c 0} c_{0}+f_{2 f 0} f_{0}+f_{2 g 0} g_{0} \\
+f_{2 a 1} a_{1}+f_{2 b 1} b_{1}+f_{2 c 1} c_{1}+f_{2 f 1} f_{1}+f_{2 g 1} g_{1}-f_{2 X} \Delta X-f_{2 Y} \Delta Y-f_{2 Z} \Delta-l_{2}
\end{array}\right.
$$

Where, $f$.. are coefficient of error equation about refined orbit and attitude model parameters and unknown ground point coordinates increment. $l x 、 l y$ for the constant term. Based on R-Cp geometric imaging model error equations of pixel coordinates, ground coordinate, refined model coefficient of ephemeris and attitude are got for adjustment model of satellite SAR images positioning: 


$$
\left\{\begin{array}{rr}
V_{x y}=B_{g} g+B_{\mathrm{t}} t-L_{x y} \cdots \ldots . . P_{x y} \\
V_{g}=E_{g} g \quad-L_{g} \ldots \ldots . . P_{g} \\
V_{t}=\quad E_{\mathrm{t}} t-L_{t} \cdots \cdots \cdots . . P_{t}
\end{array}\right.
$$

Where, $B_{g}=\left[-f_{X s},-f_{Y S},-f_{Z s}\right]^{\mathrm{T}}$ is the unknown coefficient matrix of ground point increment, $B_{t}=\left[f_{i a 0}, f_{i a 1}, f_{i b 0}, f_{i b 1}, f_{i c 0}, f_{i c 1}, f_{i f 0}, f_{i f 1}\right.$, $\left.f_{i g 0}, f_{i g 1}\right]^{\mathrm{T}}(i=1,2)$ is the unknown coefficient matrix of the refined model parameters;

$\boldsymbol{L}$ is the constant vector, which $L_{x y}=\left[0-x_{0}, y-y_{0}\right]$ is the residuals for image coordinate observation value, $L_{\mathrm{g}}=\left[X-X_{0}, Y-Y_{0}, Z-Z_{0}\right]^{\mathrm{T}}$ is the residuals for ground coordinate observation value;

$x^{0}, y^{0}$ is the calculated image coordinate based on the initial value of unknown parameters, $\left[X^{0}, Y^{0}, Z^{0}\right]^{\mathrm{T}}$ for the initial approximation or adjustment iterative approximation value;

$g:[\Delta X, \Delta Y, \triangle Z]^{\mathrm{T}}$ and $t:\left[a_{0}, a_{1}, b_{0}, b_{1}, c_{0}, c_{1}, f_{0}, f_{1}, g_{0}, g_{1}\right]^{\mathrm{T}}$ represent the increment of ground control point coordinates, refined ephemeris model parameters and refined attitude model parameters;

$P_{x y}, P g 、 P t$ are the model parameters weight matrix of image coordinates, the ground point coordinates, and ephemeris attitude observation drift model parameters, which are calculated by corresponding accuracy of observation values. The accuracy of refined parameters $\left(m_{\mathrm{a} 0}, m_{\mathrm{b} 0}, m_{\mathrm{c} 0}\right)$ consistent with the accuracy of the satellite orbit surveying, $\left(m_{\mathrm{a} 1}, m_{\mathrm{b} 1}, m_{\mathrm{c} 1}\right)$ consistent with the accuracy of satellite speed, $\mathrm{m}_{f 0}, \mathrm{~m}_{\mathrm{g} 0}$ consistent with the attitude measurement and control accuracy, $\mathrm{m}_{f 1}, \mathrm{~m}_{g 1}$ with the same accuracy to the attitude stability(change rate).

The ground point coordinates and the parameters of refined orbit attitude model could be solved by least squares.

\section{TERRASAR STEREO POSITIONING TEST}

\subsection{Test data}

Experiment with TerraSAR-X satellite images, TerraSAR is a new commercial high-resolution radar satellite for earth exploration, which manufacturing joint by the German Space Agency and the European Astor company, moving in the sun synchronous orbit with $514 \mathrm{~km}$ altitude and 98 degrees inclination, and revisit cycle of 11 days. The sensor has a spatial resolution down to $1 \mathrm{~m}$ and operates in Stripmap, Spotlight, and ScanSAR modes with selectable or dual polarization (Buckreuss, 2003, Schwerdt, 2005).

Stereo positioning respectively adopt the same side-looking direction stereo images and opposite side-looking direction stereo images to test, the same side-looking direction stereo positioning refer to the ground intersection point on one side of two radar sensors site, while the opposite side looking direction stereo positioning mean the ground intersection point between the two sensors site. The length of same side-looking direction stereo baseline or the forward intersection angle is usually smaller than that of opposite direction.

TerraSAR-X same side-looking direction stereo images got in October 2009, which located in the steepness Mountains in western China, the elevation of the measured area is at $3500 \mathrm{~m}$ $5500 \mathrm{~m}$, and the geographical coordinate of center area is $\left(32.25^{\circ}, 95.93^{\circ}\right)$,the sample resolution in azimuth direction respectively and in slant range direction respectively are $(2.18 \mathrm{~m}, 1.36 \mathrm{~m}),(2.34 \mathrm{~m}, 1.36 \mathrm{~m})$.the two scenes are as shown in Figure (3).
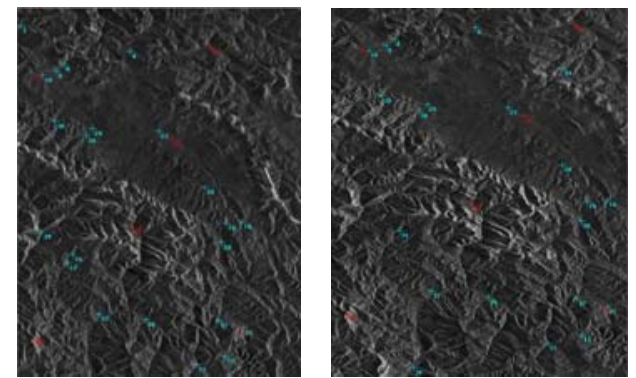

Figure 3. the same side-looking direction stereo images

The base-height ratio between two images is 0.078 , the forward intersection angle between the homologous and corresponding ground points is about 12 degree, and the two image overlap area greater than $80 \%$, in this area totally have 22 known coordinates ground points, the relationship between image and the ground point distribution shown in Figure (4).

TerraSAR-X opposite side-looking direction stereo images got in July 2009, the geographical coordinate of image center area is $(31.15,94.32)$. the sample resolution in azimuth direction and in slant range direction for the two SAR images respectively are $(2.03 \mathrm{~m}, 1.36 \mathrm{~m}),(1.58 \mathrm{~m}, 1.36 \mathrm{~m})$. The elevation of surveying area is at $3800 \mathrm{~m}-5600 \mathrm{~m}$, the base-height ratio is 1.27 , and the forward intersection angle of ground point is 67 degree, images overlap area within the known geographical coordinates points are total of 12 , the relationship between the two scene images and ground points distribution shown in Figure (5) below.
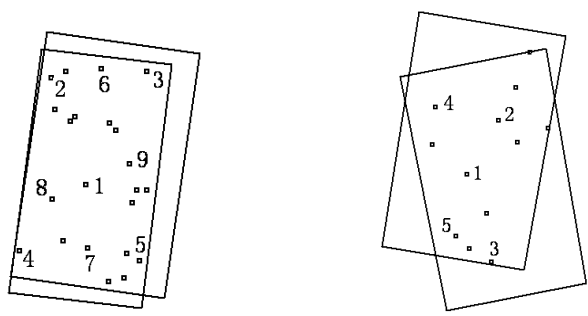

Fig4. the distribution of same Fig5. the distribution of opposite side-looking direction stereo side-looking direction stereo images and ground points images and ground points

Ground known points available through triangulation encryption methods by optical SPOT5 HRS and HRG images with Integration Mapping Software PixelGrid (Zhang, 2009), and the precision higher than the accuracy of 1:50 000 topographic maps.

\subsection{Test and Test analysis}

(1) TerraSAR imaging compensated attitudes 
Attitudes oscillation affects SAR imaging same with the track oscillation. In this paper, every TerraSAR-X image photographic time on the orbit is 8 seconds, and the original attitude value and the calculated attitude value based doppler 0 value within this time were extracted. Plot the attitude change curve within photographic time for a scene image as Figure (6) shows. Among them, the left diagram for the roll angle sketch map, and the right picture for yaw angle sketch map. In sketch map, the variation curves represent the original measured values, smooth curves for the motion-compensated reference value.
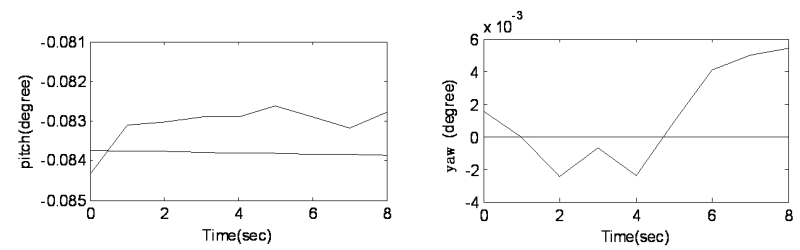

Figure 6 sketch map for attitude measured value and compensation value

\section{(2) Stereo positioning test with same side-looking direction images}

Tests through select different numbers of ground points as control point, the number separate is 0,1 (No.1), 4(No.2-No.5), 9(No.1-No.9) and 15(uniform distribution), at the same time, the remaining points are taken as check points for stereo positioning accuracy test. Transform the adjustment value and measured value of ground points to Gauss projection coordinate system, and calculate the error based the adjustment value and measured value, then compute the plane and height accuracy for control points and check points .

For comparison, we based the R-D model and with the satellite orbit one order linear refined model parameters as orientation parameters, the speed change value calculated according to the position vector of the time derivative, as formula (11).

$$
\vec{V}=\vec{P} / d t+d \overrightarrow{\mathrm{P}} / d t=V_{0}+d V
$$

The equation (9) was instead by error equation that established base Doppler condition, and re-establish the stereo positioning model based on R-D model and experiment. Table (1) statistics of R-Cp model and R-D model for stereo positioning accuracy, include the plane and elevation accuracy for control points and check points, and the plane and elevation coordinate maximum error of checkpoint.

\section{(3)Stereo positioning test with opposite side-looking direction images}

In this test, we also select different numbers of control point to experiment, there are 1 GCP (No.1), 2 GCPs (No.2, 5), 5 GCPs (No1-5) and 11 GCPs (LOOCV assess precision method, (Brovelli M, et al., 2006)), and take the R-Cp model as the foundation for stereo positioning test, then get the plane and elevation precision for control points and check points, and the maximum error, statistics in Table (2).
Table 1 stereo positioning accuracy statistics with same sidelooking direction images

\begin{tabular}{|c|c|c|c|c|c|c|c|c|c|}
\hline \multicolumn{4}{|c|}{ Number of GCPs } & 0 & 1 & 2 & 4 & 9 & 15 \\
\hline \multirow{8}{*}{$\begin{array}{l}\text { Ground } \\
\text { Points } \\
\text { Precision } \\
\text { And } \\
\text { Error }\end{array}$} & \multirow{6}{*}{$\begin{array}{l}\mathrm{R}-\mathrm{Cp} \\
\text { model }\end{array}$} & \multirow[t]{2}{*}{ GCPs } & $\mathrm{m}_{\mathrm{p}}$ & & 0.5 & 2.5 & 6.2 & 7.6 & 7.8 \\
\hline & & & $\mathrm{m}_{\mathrm{H}}$ & & 0.8 & 1.9 & 3.5 & 4.3 & 4.6 \\
\hline & & \multirow[t]{2}{*}{$\mathrm{CPs}$} & $m_{p}$ & 29.8 & 12.7 & 11.1 & 10.4 & 9.1 & 8.9 \\
\hline & & & $\mathrm{m}_{\mathrm{H}}$ & 13.2 & 8.3 & 7.7 & 5.8 & 5.3 & 5.1 \\
\hline & & \multirow{2}{*}{$\begin{array}{l}\text { Max } \\
\text { Errors }\end{array}$} & $V_{p}$ & 42.7 & 19.4 & 18.5 & 18.1 & 16.5 & 16.2 \\
\hline & & & $\mathrm{V}_{\mathrm{H}}$ & 23.4 & 15.0 & 13.3 & 9.9 & 9.6 & 9.0 \\
\hline & \multirow{6}{*}{$\begin{array}{l}\text { R-D } \\
\text { model }\end{array}$} & \multirow[t]{2}{*}{ GCPs } & $\mathrm{m}_{\mathrm{p}}$ & & 0.6 & 2.6 & 6.4 & 8.0 & 8.3 \\
\hline & & & $\mathrm{m}_{\mathrm{H}}$ & & 1.0 & 2.0 & 3.7 & 4.5 & 4.9 \\
\hline \multirow{4}{*}{ (m) } & & \multirow[t]{2}{*}{ CPs } & $\mathrm{m}_{\mathrm{p}}$ & 29.7 & 12.6 & 11.1 & 10.5 & 9.3 & 9.2 \\
\hline & & & $\mathrm{m}_{\mathrm{H}}$ & 13.2 & 8.3 & 7.7 & 5.8 & 5.4 & 5.2 \\
\hline & & \multirow{2}{*}{$\begin{array}{l}\text { Max } \\
\text { Errors }\end{array}$} & $V_{P}$ & 42.5 & 19.2 & 18.4 & 18.2 & 16.7 & 16.5 \\
\hline & & & $\mathrm{V}_{\mathrm{H}}$ & 23.3 & 14.9 & 13.2 & 9.9 & 9.7 & 9.1 \\
\hline
\end{tabular}

Table 2. stereo positioning accuracy statistics with reverse side looking direction images

\begin{tabular}{|l|l|l|l|l|l|l|l|}
\hline \multicolumn{3}{|c|}{ Number of GCPs } & 0 & 1 & 2 & 5 & 11 \\
\hline \multirow{2}{*}{$\begin{array}{l}\text { RMSE } \\
\text { and }\end{array}$} & $\begin{array}{l}\text { GCPs } \\
\text { Error } \\
(\mathrm{m})\end{array}$ & $\mathrm{P}$ & & 0.8 & 3.3 & 6.1 & 6.7 \\
\cline { 2 - 8 } & $(\mathrm{RMSE})$ & $\mathrm{H}$ & & 0.9 & 2.7 & 3.8 & 4.0 \\
\cline { 2 - 8 } & CPs & $\mathrm{P}$ & $\mathbf{1 7 . 9}$ & $\mathbf{1 0 . 3}$ & $\mathbf{9 . 9}$ & $\mathbf{8 . 8}$ & $\mathbf{8 . 4}$ \\
\cline { 2 - 8 } & RMSE) & $\mathrm{H}$ & $\mathbf{2 9 . 2}$ & $\mathbf{6 . 1}$ & $\mathbf{5 . 5}$ & $\mathbf{4 . 7}$ & $\mathbf{4 . 5}$ \\
\cline { 2 - 8 } & $\begin{array}{l}\text { Max } \\
\end{array}$ & $\mathrm{P}$ & 33.6 & 17.7 & 16.1 & 14.0 & 13.3 \\
\cline { 2 - 8 } & Errors & $\mathrm{H}$ & 35.9 & 11.2 & 9.8 & 9.3 & 9.1 \\
\hline
\end{tabular}

\subsection{Test analysis}

(1) The attitude value calculated with 0 Doppler conditions show that the referenced attitude of SAR imaging has been compensated, attitude compensation is also part of the SAR imaging motion compensation. TerraSAR-X compensating referenced attitude and the actual measured value is very close, but the attitude shock has been eliminated.

(2) With the compensated attitude as initial value, the positioning result used R-Cp model is close to R-D model. TerraSAR-X compensating referenced attitude is in the vicinity of the measured attitude, when adjustment can take the middle values or mean values of measurement as the value of attitude, it only has a smaller system error with the actual motion compensation referenced value. In the case of one or more control point, it would be easily to eliminate.

(3) In the absence of the ground control points, the plane accuracy of TerraSAR-X image with same and opposite direction observation separately are $29.8 \mathrm{~m}$ and $17.9 \mathrm{~m}$, the elevation precision are $13.2 \mathrm{~m}$ and $29.2 \mathrm{~m}$, the result show that location accuracy differences of this two kinds methods are significant. In the condition of few amount ground control points, the two kinds of images stereo positioning precision are improved significantly, with the number of the control points increased, plane and elevation precision are also improved.

(4) Along with the control points increased, the accuracy improved rate of the opposite side-looking direction images is less than the improved rate of same looking direction images, 
but with the same number of control points, the check points precision of stereo positioning for the opposite looking direction images is higher than the precision of same looking direction images. There is a close relationship between the stereo positioning accuracy and base-height ratio, and with the base-height ratio is greater, the positioning accuracy is higher and more stability.

The control points in test are photogrammetric for SPOTHRS/HRG optical images, relative to the TerraSAR-X image resolution that the precision is still a bit low, which would affect the accuracy and reliability of the orientation parameters solution. In the condition with higher precision measured GCPs, TerraSAR-X stereo positioning accuracy in theoretical will be better than test results in paper.

\section{CONCLUSION}

The R-Cp geometric imaging model which take exterior orientation elements as orientation parameters could be used as the SAR image 3D positioning, and this method can directly adopt the combined adjustment methods developed by optical images, which show that the R-Cp geometric imaging model has certain advantages in the side-looking radar digital photography process, the rigorous stereo positioning model for satellite TerraSAR-X images that can satisfy the mapping accuracy in mountainous terrain. The surveyed area tests showed that there was a big relationship between the positioning accuracy and the number of ground control points and distribution, the coordinate measurement accuracy, and the size of the forward intersection angle of the ground point.

In some steepness mountain area that snow covered all the year round and no one could arrive, it is difficult to only use optical images to map, but the swath of SPOT5HRS images is much larger than the TerraSAR-X high resolution image, this resulted that use sparse known ground control points in SPOT5HRS/HRG could achieve many three-dimensional triangulation encryption points at gaps of snow covering for large area. We can take advantage of these encryption points for the TerraSAR-X image stereo positioning and mapping, and decrease field work for control points surveying. This method provides an effective choice for complete the 1:50,000 topographic mapping of China western snow-capped mountains region, also the model built in this paper has been used in 1:50000 mapping project for China western area.

\section{ACKNOWLEDGEMENTS}

This paper is supported by National Natural Science Foundation of China( No. 41071237 ), the National High Technology Research and Development program of China (863 program) (No. 2011AA120401, No. 2011AA120402).

\section{REFERENCES}

Brovelli M A, Crespi M, Fratarcangeli F, Giannone F and Realini E. 2006. Accuracy Assessment of High Resolution Satellite Imagery by Leave-one-out method. Proceedings of the 7th International Symposium on Spatial Accuracy Assessment in Natural Resources and Environmental Sciences, July, Lisbon, Portugal: 533-542.

Buckreuss, S., W. Balzer, P. Muhlbauer, R. Werninghaus, and W. Pitz, 2003: The TerraSAR-X satellite project. Proc. IEEE Int.Geoscience and Remote Sensing Symp. (IGARSS03), Toulouse,France, IEEE, 3096-3098.

Cheng C Q, Zhang J X, Deng K Z and Zhang L . 2012. RangeCoplanarity equation for radar geometric imaging. Journal of Remote Sensing, 16(1): 38-49.

Cheng C Q, Zhang J X.2012. Rigorous Positioning with Sidelooking Radar Imagery Based on Range-Coplanarity Model. Journal of Remote Sensing, 16(5) (waiting for publishing).

Gelautz M, Frick H, Raggam J, Burgstaller J and Leberla F. 1998. SAR image simulation and analysis of alpine terrain. ISPRS Journal of Photogrammetry and Remote Sensing, 53(1): $17-38$.

Johnsen H, Lauknes L and Guneriussen T. 1995. Geocoding of fast-delivery ERS-1 SAR image mode product using DEM data. International Journal of Remote Sensing, 16(11): 1957-1968.

Konecny G and Schuhr W. 1988. Reliability of Radar Image Data. 16th ISPRS, (16): 92-101.

La Prade G., 1963. An analytical and experimental study of stereo for radar. Photogrammetric Engineering, 29 (2), 294-300. Lauknes I, E. Malnes, “Automatical geocoding of Envisat ASAR products,” In: Proceed-ings of the 2004 Envisat \& ERS Symposium, Salzburg, Austria, 6-10 September 2004, ESA SP572, April 2005.

Leberl F. 1978. Radargrammetry for Image Interpretation. ITC Technical Report.

Leberl F. 1990. Radar image grammetric processing. Artech House.

Schwerdt, M., D. Hounam, J.-L. Alvarez-Pères, and T. Molkenthin,2005: The calibration concept of TerraSAR-X: A multiple-mode, high-resolution SAR. Can. J. Remote Sens., 31,30-36.

Zhang L, Zhang J X, and Chen X Y. 2009.Block adjustment with SPOT-5 imagery and sparse GCPs based on RFM[J]. Acta Geodaetica et Cartographica Sinica, 38(4):P302310 (in Chinese) . 